\title{
The effect of neurosphere culture conditions on the cellular metabolism of glioma cells
}

\author{
Ulf Dietrich Kahlert' ${ }^{1,2}$, Katharina Koch ${ }^{2}$, Abigail Kora Suwala², Rudolf Hartmann ${ }^{3}$, Menglin Cheng ${ }^{4}$, \\ Donata Maciaczyk ${ }^{2}$, Dieter Willbold ${ }^{3,5}$, Charles G. Eberhart ${ }^{1}$, Kristine Glunde ${ }^{4}$, Jarek Maciaczyk ${ }^{2}$ \\ ${ }^{1}$ Department of Pathology, Division of Neuropathology, Johns Hopkins Hospital, Baltimore, USA, 2Department of Neurosurgery, \\ University Medical Center Düsseldorf, Germany, ${ }^{3}$ Institute of Complex Systems ICS-6, Research Center Jülich, Germany, \\ ${ }^{4}$ Division of Cancer Imaging Research, Russell H. Morgan Department of Radiology and Radiological Science, Johns Hopkins \\ Hospital, Baltimore, USA, ${ }^{5}$ Institute of Physical Biology, Heinrich-Heine-University Düsseldorf, Germany
}

\begin{abstract}
Malignant gliomas, with an average survival time of 16-19 months after initial diagnosis, account for one of the most lethal tumours overall. Current standards in patient care provide only unsatisfying strategies in diagnostic and treatment for high-grade gliomas. Here we describe metabolic phenomena in the choline and glycine network associated with stem cell culture conditions in the classical glioma cell line U87. Using high-resolution proton magnetic resonance spectroscopy of cell culture metabolic extracts we compare the metabolic composition of U87 chronically propagated as adherent culture in medium supplemented with serum to serum-free neurosphere growth. We found that the switch to neurosphere growth, besides the increase of cells expressing the putative glioma stem cell marker CD133, modulated a number of intracellular metabolites including choline, creatine, glycine, and myo-inositol that have been previously reported as potential diagnostic markers in various tumours.

These findings highlight the critical influence of culture conditions on glioma cell metabolism, and therefore particular caution should be drawn to the use of in vitro system research in order to investigate cancer metabolism.
\end{abstract}

Key words: glioma, metabolism, neurosphere, CD133, choline, creatine, glycine, myo-inositol.

\section{Introduction}

Glioblastoma (GBM) is the most common and lethal adult glial brain tumour, with a mean overall survival of 16-19 months after primary diagnosis under the current standard-of-care treatment scheme [26]. Despite enormous research efforts towards early diagnosis and more efficient treatment, the prognosis of GBMs remains dismal.

The influence of culture conditions has been widely investigated in the field of glioma research, suggest- ing that neurosphere cultures, compared to adherent growth, more closely resemble the original patient's tumour [29] showing high stem cell compartment [1] and therefore are more suitable for testing of novel therapeutic spectras approaches [30]. In this report we describe altered relative concentrations of the cholines, creatine, myo-inositol, and glycine in the human GBM cell line U87 propagated under stem cell conditions as compared to classical monolayer culture. Furthermore, U87 neurospheres showed significant high- 
er levels of the putative GBM stem cell marker CD133 as their serum-propagated counterparts. Detection and targeting of miss-regulated choline-, myo-inositol-, creatine-, and glycine-metabolism has been described to have potential utility in the diagnosis and treatment of malignant gliomas $[2-4,13,16,19]$.

This is, to our knowledge, the hitherto first link of changes in those oncometabolites $[4,25]$ to variations in cell culture conditions of glioma cells. Interspectral co-analysis of metabolite concentrations under the two propagation conditions identified reductions in ratios of phosphocholine to glycerophosphocholine (PC/GPC) and glycine to total choline (Gly/tCho) but increases in the quotient of total choline to total creatine (tCho/tCre) and PC/tCre, as well as Gly/myo-inositol (Gly/myo). This work should draw the attention of the scientific community on possible in vitro artefacts and on the need for appropriate models most closely resembling the in vivo biology of investigated tumours.

\section{Material and methods}

\section{Cell culture}

U87 cell line was purchased from American Type Cell Culture bank (www.ATCC.com) and propagated either as adherent culture in Dulbecco's modified Eagle's medium (DMEM, Life Technologies) containing $20 \%$ foetal calf serum or as neurospheres (stem cell culture) in DMEM/F12 (3:1, both Life Technologies) medium supplemented with B27 (Life Technologies), $20 \mathrm{ng} / \mathrm{ml}$ recombinant human basic fibroblast growth factor (bFGF, Peprotech), $20 \mathrm{ng} / \mathrm{ml}$ recombinant human epidermal growth factor (EGF, Peprotech), Anti-Anti (Life Technologies), and $5 \mu \mathrm{g} / \mathrm{ml}$ heparin (Sigma-Aldrich), as described before [12]. Cells were passaged at least eight times in each culture condition before they were subjected to experimental analysis.

Cell line identity was confirmed by analysis of nine tandem repeats plus a gender-determining marker, Amelogenin, using the StemElite kit (Promega, Supporting Information File).

\section{Metabolic extractions of in vitro cultures, proton nuclear magnetic resonance spectroscopy $\left({ }^{1} \mathrm{H}-\mathrm{NMR}\right)$ and metabolite quantification.}

A minimum of $7 \times 10^{6}$ cells were collected for each extraction (each condition $n=3$ ). A methanol-chlo- roform-water (1/1/1, v/v/v) dual phase cell lyses protocol was applied to extract water and lipid-soluble metabolites as described before [9]. In this study we only assessed content of the water-soluble extracts. The lyophilised water-soluble extracts were resolved in $20 \mathrm{mM}$ phosphate buffer $\mathrm{pH} 7\left(10 \% \mathrm{v} / \mathrm{v} \mathrm{D}_{2} \mathrm{O}\right.$, Sigma-Aldrich, MO, USA) supplemented with sodium (2,2-dimethyl-2-silapentane-5-sulfonate) (DSS; Euriso-top, internal NMR-standard, STD) for the scans at the Research Centre in Jülich (Germany), and in $\mathrm{D}_{2} \mathrm{O}$ containing $0.24^{*} \mu \mathrm{mol} 3$-(trimethylsilyl) propionic2,2,3,3,- $\mathrm{d}_{4}$ acid (Sigma-Aldrich, internal concentration standard, STD) at John Hopkins Hospital. The spectras were acquired at $25^{\circ} \mathrm{C}$ on a Bruker Avance 500 spectrometer operating at $11.7 \mathrm{~T}$ using a $5-\mathrm{mm} \mathrm{HX}$ inverse probe, as previously described [9], at the Department of Radiology in the John Hopkins Hospital, and a Bruker Avance III HD 600 spectrometer operating at $14.1 \mathrm{~T}$ using a $5-\mathrm{mm}$ TXI-cryo-probe at the Institute of Complex Systems (ICS-6) in the Research Centre in Jülich (Germany). Water suppression was achieved by applying a pulse-sequence using excitation sculpting with gradients. The fully relaxed $1 \mathrm{H}-\mathrm{NMR}$ data were processed, and the signal integrals listed below were measured using Mestrenova V10 software (Mestrelab). All metabolite concentrations were quantified through peak integration after standardised performed phase correction and baseline fitting. The respective concentration standard served as intra-spectral normalisation for each $1 \mathrm{H}-\mathrm{NMR}$ spectra, and metabolite concentrations within the same spectra were co-analysed and presented as means plus SDs.

\section{FACS-based assessment of CD133+ cell population}

Cells were stained for cell surface antigen CD133 (AC133-PE, \#130-098-826, Miltenyi Biotec) according to the manufacturer's protocols, as described before [11]. AC133-antibody without a fluorescent dye (AC133-pure, \#130-090-422, Miltenyi Biotec) was used as control. Fluorescence activated cell sorting (FACS) was performed on an Accuri C6 cytometer (BD Biosciences, Franklin Lakes, NJ) whereas for post-processing data analysis FlowJo v10 software (Flowjo, Tree Star Inc, Ashland, OR) was applied.

\section{Cell apoptosis assays}

Cultures were dissociated to single cell suspension and viable cells were quantified using the MUSE 
Count \& Viability Assay Kit (\#MCH100102, Merck KGaA, Darmstadt on a Muse Cell Analyser [\#05003115, Merck KGaA]). Apoptotic cells were quantified using the AnnexinV \& Dead Cell Kit (\#MCH100105, Merck KGaA, Darmstadt, Germany) on the Muse Cell Analyser, according to the manufacturer's protocol. A minimum of 2000 gated events were acquired.

\section{Statistical evaluation}

Statistical evaluation was performed using Students $t$-test in Statistica (Statsoft, OK, USA) and presented as means plus respective standard deviations.

\section{Results}

Modification of the culture condition alters the relative concentration of choline, creatine glycine, and myo-inositol in U87 glioma cells.

High-resolution ${ }^{1} \mathrm{H}-\mathrm{NMR}$ spectra were obtained as described above, and a representative spectrum is shown in Figure $1 \mathrm{~A}$. The most prominent metabolites were annotated and quantified, including adenine (Ade), myo-inositol (myo), glycine (Gly), phosphocholine (PC), glycerophosphocholine (GPC), free choline (fCho), total choline (tCho $=\mathrm{fCho}+\mathrm{PC}+\mathrm{GPC}$ ), total creatine (tCre), glutathione (GSH), glutamine (Gln), glutamate (Glu), N-acetylaspartylglutamate (NAAG), alanine (Ala), lactate (Lac), threonine (Thr), and valine/isoleucine (Val//so). Due to the previously described importance in glioma biology we focused our quantitative analysis on choline, creatine, glycine, and myo-inositol ratios (Fig. 1B).

The switch to neurosphere media decreased the ratio of PC/GPC (40\%) and significantly diminished the Gly/tCho concentration (70\%). In contrast, we detected an increase in the ratios of Gly/myo (65\%), tCho/tCre (300\%), and PC/tCre (280\%), reaching statistical significance for the latter two.

\section{Neurosphere assay condition increases the expression of cell surface marker CD133}

U87 culture in classical serum-containing growth medium expressed very low levels of CD $133^{+}$cells (about the amount of the negative control, 1\% CD133+ cells on average, Fig. 1C). Following their transfer into stem cell conditions, the CD133- population was increased to $18.5 \%$ ( $p$-value $\leq 0.001$, Fig. $2 A$ ). Similar results of $\mathrm{CD}_{133^{+}} \mathrm{U} 87$ cells in free-floating culture conditions have been published previously $[6,21]$.

\section{U87 neurosphere cultures exhibit a higher fraction of apoptotic cells}

We identified increased levels of apoptosis and cell death in cells propagated under neurosphere growth conditions compared to their adherent counterparts as assessed by AnnexinV/Propiodium iodide-based quantification (Fig. 2B).

\section{Discussion}

In this work we analysed the changes of U87 cell metabolism depending on whether a monolayer or 3D in vitro propagation has been applied. Our study of this human glioma cell line confirms the accumulation or preferential selection of $\mathrm{CD}_{133^{+}}$cells, a putative brain tumour stem cell marker [15], under prolonged stem cell culture conditions compared to propagation in serum-containing media. Recent studies addressing the effect of different culture conditions in in vitro mouse models of GBM described the robust enhancement of stem cell marker expression and self-renewal capacity of cells transferred from adherent growth to spheroid culture [1]. Moreover, neurosphere cultures are suggested to more closely resemble the original patient's tumour [29] and therefore are more suitable for testing novel therapeutic approaches [30].

Using high-resolution magnetic resonance spectroscopy $\left({ }^{1} \mathrm{H}-\mathrm{NMR}\right)$ in extracts of cells chronically propagated under serum-free neurosphere growth, we identified several alterations in metabolite concentrations with proposed diagnostic utility as a response to changed growth stimuli.

Phosphocholine (PC), glycerophosphocholine (GPC), and free choline (fCho) all play an important role in the membrane phospholipid household that accompanies cell cycle progression [10]. Moreover, dysregulated choline metabolism has already been proposed as an underlying molecular event during cancerous malformation in a variety of cancers [7], including GBM [2]. Interestingly, we found that U87 glioma neurospheres exhibit a reduced PC/GPC ratio, although it is not statistically significant. The PC/GPC ratio has been shown to be elevated in aggressive breast cancer cells [8], however, the diagnostic impact of this parameter in brain cancers has been challenged [18]. The tCho/tCre quotient is a historically established diagnostic biomarker in brain tumours, which increases with progression of malignancy $[20,23,24]$. Concordant with this data we revealed that the tCho/ 


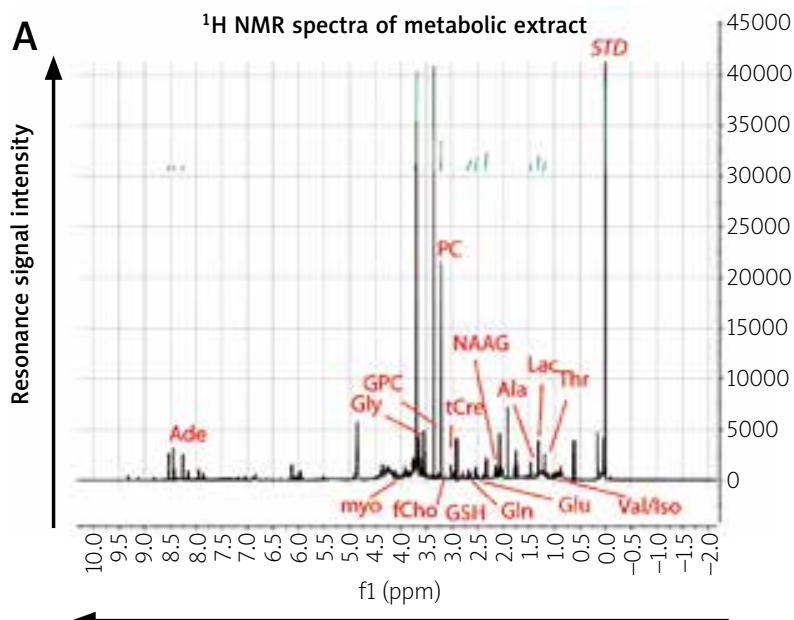

B

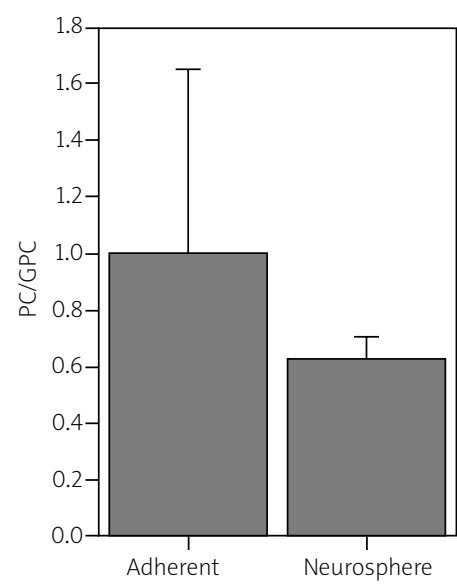

Chemical shift [ppm]
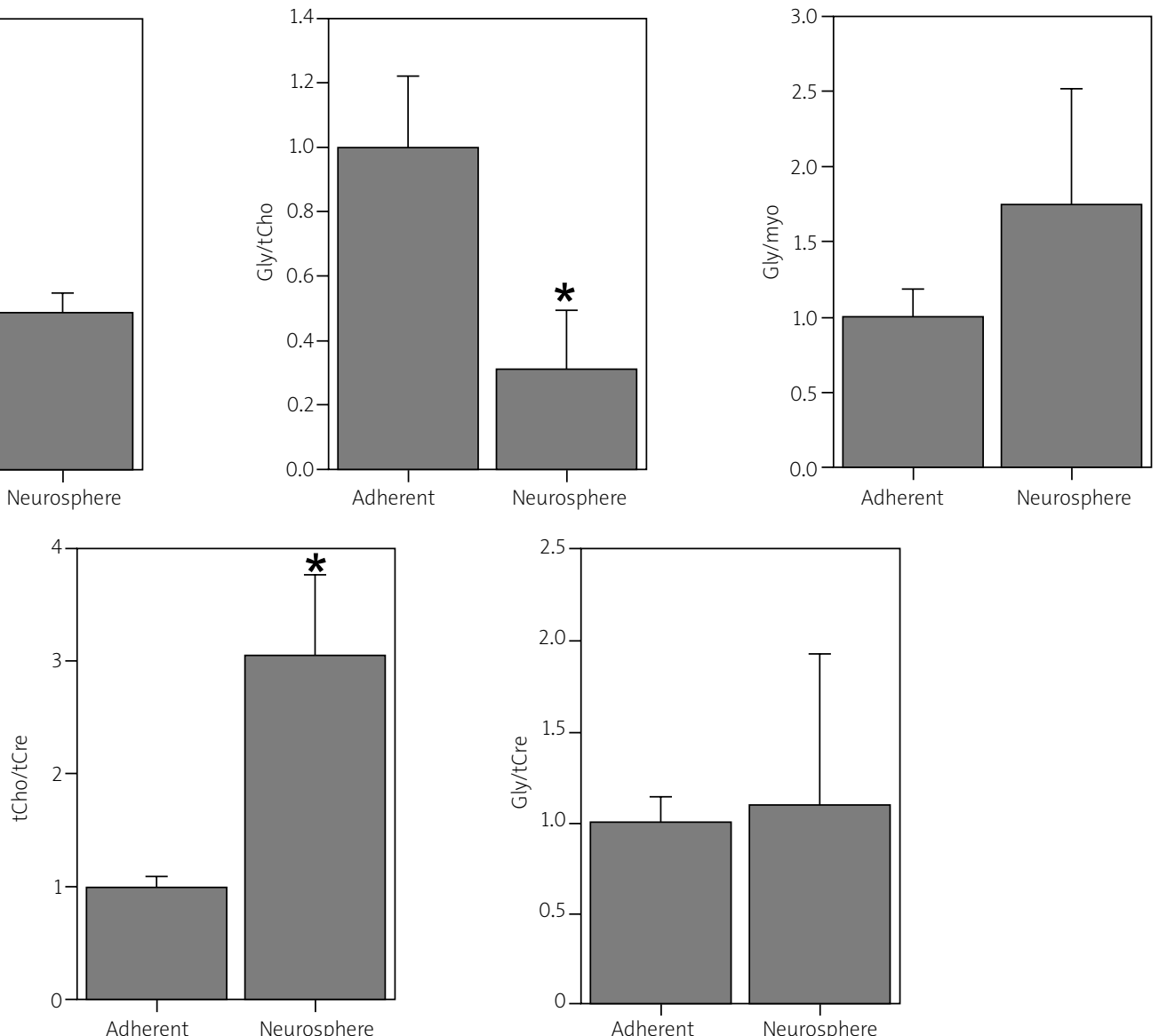

Fig. 1. Global metabolic profiling of U87 glioma cells under adherent and suspension growth: Representative ${ }^{1} \mathrm{H}$ NMR spectra of the intracellular metabolites of $U 87$ glioma cells with annotated adenine (Ade), myo-inositol (myo), glycine (Gly), phosphocholine (PC), glycerophosphocholine (GPC), free choline (fCho), total choline (tCho $=f C h o+P C+G P C)$, total creatine (tCre), glutathione (GSH), glutamine (Gln), glutamate (Glu), N-acetylaspartylglutamate (NAAG), alanine (Ala), lactate (Lac), threonine (Thr) as well as valine/isoleucine (Val/Iso) plus internal concentration standard (STD). A) Relative quantification of phosphocholine, glycerophosphocholine, glycine, creatine, and myo-inositol revealed altered metabolism after transfer of U87 cells into neurosphere growth conditions: reduction of PC/GPC and Gly/tCho whereas Gly/myo and tCho/tCre ratios were increased (B), $p=0.05$. 

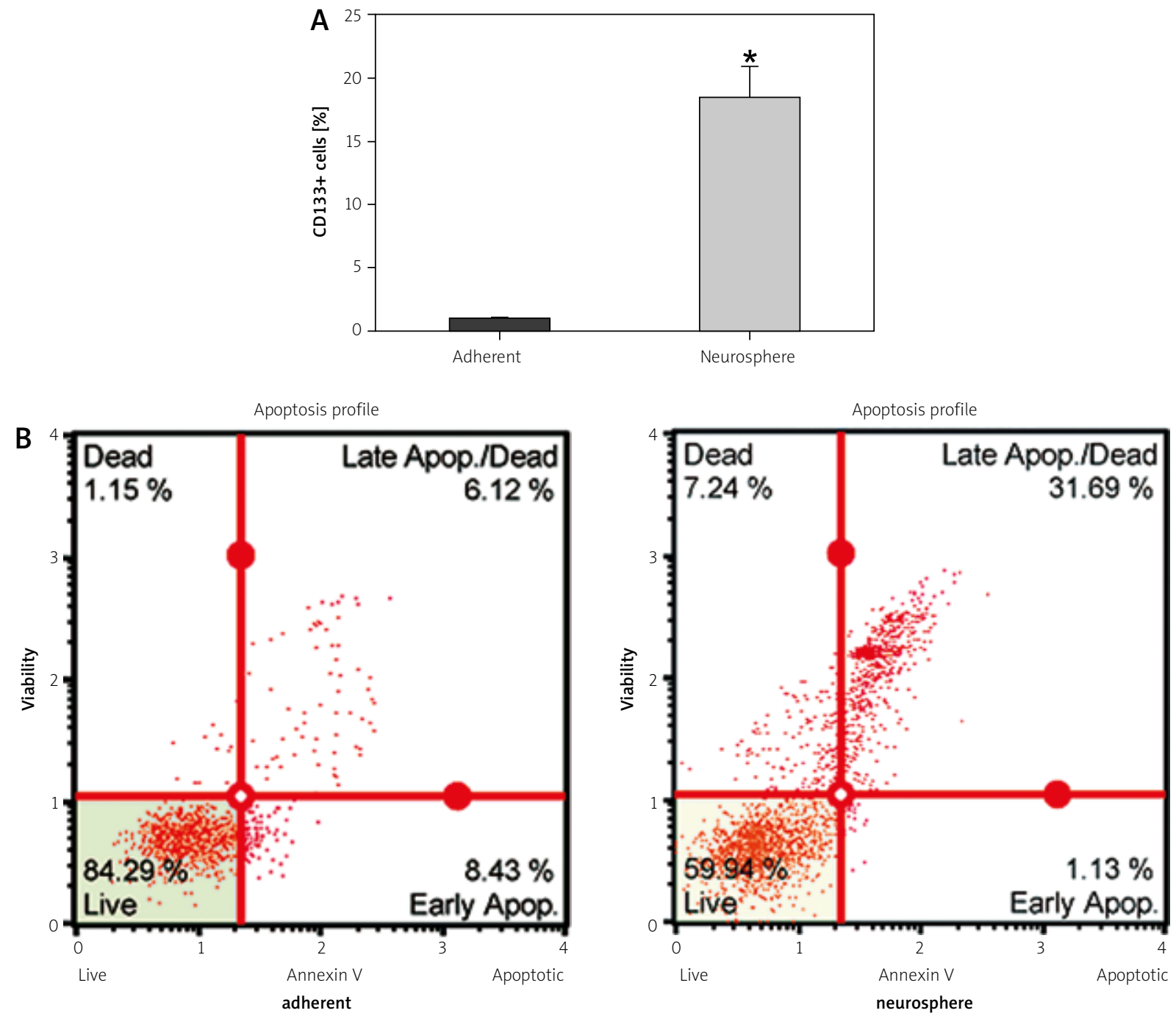

Fig. 2. U87 neurosphere cells compared to monolayer cells show an increased population of cells expressing the cell surface marker CD133 (A) ( $p$-value $\leq 0.001)$ and are characterised by a higher number of apoptotic cells (B).

tCre ratio is significantly increased in cells cultivated as neurospheres.

Glycine (Gly), a currently intensively studied metabolite with oncogenic potential, has been reported to be directly correlated with glioma malignancy [4]. Hypoxic glioma cells, which are known to contain a population of cells with high stem-like signature [11], are highly susceptible to glycine metabolism [13]. In addition, efforts using ${ }^{1} \mathrm{H}-\mathrm{NMR}$-spectroscopy to grade brain tumour malignancy based on their cellular metabolism suggested glycine as a negative prognostic biomarker $[5,17,22]$. The Gly/tCre ratio is thought to be a suitable parameter for grading of gliomas and for their distinction from brain metastasis [14]. Interestingly, we did not notice differenc- es in Gly/tCre between the two growth conditions. Therefore, we performed co-analyses with additional metabolites, including myo-inositol (myo) a metabolite reported to be reduced in high-grade as compared to low-grade brain tumours $[3,19]$. We could detect that the Gly/myo ratio, reported to be a valid marker for high-grade gliomas including GBM [22], is increased in suspension cells, indicating either the increase of Gly or the reduction of myo in neurospheres, as compared to adherent cells. An important route for the synthesis of glycine involves the degradation of choline [28]. We therefore sought to compare their relative concentrations in the two culture conditions and found the Gly/tCho ratio significantly diminished (about $75 \%$ decrease) 
in neurosphere cells. The reduction of Gly/tCho could potentially be due to elevated concentrations of tCho in stem-like neurospheres, as increased tCho is an accepted marker for high-grade brain tumour malignancy [27].

\section{Conclusions}

In summary, we conclude that switching the cell culture conditions for U87 cells effectively alters their cellular metabolism, influencing a variety of metabolites with reported importance in glioma and glioma stem cell progression. Cells propagated in suspension and adherent growth can dramatically alter their cellular proliferation and apoptosis rate due to alterations in environmental stimuli. We confirm increased apoptosis in U87 neurosphere cells compared to cells grown as a monolayer. We cannot preclude, that the described metabolic differences might, at least partially, be due to the increased cell death.

Therefore, particular caution has to be used interpreting the results of studies on cancer metabolism in the context of the in vitro model. As reported here, substantial variations in intracellular levels of metabolites might be a consequence of different culture conditions, precluding the formulation of general conclusions.

\section{Acknowledgements}

UDK is supported by the Dr. Mildred Scheel fellowship by the Deutsche Krebshilfe.

The work has been co-financed by the SFF Grant of the HHU University, Düsseldorf, Germany, awarded to JM. AKS is supported by the Friedrich-Ebert Stiftung. KK is a fellow of Düsseldorf School of Oncology (DSO) of HHU University.

\section{Disclosure}

Authors report no conflict of interest.

\section{References}

1. Ahmad M, Frei K, Willscher E, Stefanski A, Kaulich K, Roth P, Stühler K, Reifenberger G, Binder H, Weller M. How stemlike are sphere cultures from long-term cancer cell lines? Lessons from mouse glioma models. J Neuropathol Exp Neurol 2014; 73: 1062-1077.

2. Bianchi L, De Micheli E, Bricolo A, Ballini C, Fattori M, Venturi C, Pedata F, Tipton KF, Della Corte L. Extracellular levels of amino acids and choline in human high grade gliomas: an intraoperative microdialysis study. Neurochem Res 2004; 29: 325-334.
3. Castillo M, Smith JK, Kwock L. Correlation of myo-inositol levels and grading of cerebral astrocytomas. Am J Neuroradiol 2000; 21: $1645-1649$

4. Chinnaiyan P, Kensicki E, Bloom G, Prabhu A, Sarcar B, Kahali S, Eschrich S, Qu X, Forsyth P, Gillies R. The metabolomic signature of malignant glioma reflects accelerated anabolic metabolism. Cancer Res 2012; 72: 5878-5888.

5. Choi C, Ganji SK, Madan A, Hulsey KM, An Z, Zhang S, Pinho MC, Deberardinis RJ, Bachoo RM, Maher EA. In vivo detection of citrate in brain tumors by (1) $\mathrm{H}$ magnetic resonance spectroscopy at 3T. Magn Reson Med Off J Soc Magn Reson Med Soc Magn Reson Med 2014; 72: 316-323.

6. Christensen K, Aaberg-Jessen C, Andersen C, Goplen D, Bjerkvig R, Kristensen BW. Immunohistochemical expression of stem cell, endothelial cell, and chemosensitivity markers in primary glioma spheroids cultured in serum-containing and serum-free medium. Neurosurgery 2010; 66: 933-947.

7. Glunde K, Bhujwalla ZM, Ronen SM. Choline metabolism in malignant transformation. Nat Rev Cancer 2011; 11: 835-848.

8. Glunde K, Jie C, Bhujwalla ZM. Molecular causes of the aberrant choline phospholipid metabolism in breast cancer. Cancer Res 2004; 64: 4270-4276.

9. Glunde K, Shah T, Winnard PT, Raman V, Takagi T, Vesuna F, Artemov D, Bhujwalla ZM. Hypoxia regulates choline kinase expression through hypoxia-inducible factor-1 alpha signaling in a human prostate cancer model. Cancer Res 2008; 68: 172-180.

10. Jackowski S. Cell cycle regulation of membrane phospholipid metabolism. J Biol Chem 1996; 271: 20219-20222.

11. Kahlert UD, Maciaczyk D, Dai F, Claus R, Firat E, Doostkam S, Bogiel T, Carro MS, Döbrössy M, Herold-Mende C, Niedermann G, Prinz M, Nikkhah G, Maciaczyk J. Resistance to hypoxia-induced, BNIP3-mediated cell death contributes to an increase in a CD133-positive cell population in human glioblastomas in vitro. J Neuropathol Exp Neurol 2012; 71: 1086-1099.

12. Kahlert UD, Maciaczyk D, Doostkam S, Orr BA, Simons B, Bogiel T, Reithmeier T, Prinz M, Schubert J, Niedermann G, Brabletz T, Eberhart CG, Nikkhah G, Maciaczyk J. Activation of canonical WNT/ $\beta$-catenin signaling enhances in vitro motility of glioblastoma cells by activation of ZEB1 and other activators of epithelial-to-mesenchymal transition. Cancer Lett 2012; 325: 42-53.

13. Kim D, Fiske BP, Birsoy K, Freinkman E, Kami K, Possemato RL, Chudnovsky Y, Pacold ME, Chen WW, Cantor JR, Shelton LM, Gui DY, Kwon M, Ramkissoon SH, Ligon KL, Kang SW, Snuderl M, Vander Heiden MG, Sabatini DM. SHMT2 drives glioma cell survival in ischaemia but imposes a dependence on glycine clearance. Nature 2015; 520: 363-367.

14. Kinoshita Y, Kajiwara H, Yokota A, Koga Y. Proton magnetic resonance spectroscopy of astrocytic tumors: an in vitro study. Neurol Med Chir (Tokyo) 1993; 33: 350-359.

15. Li Z. CD133: a stem cell biomarker and beyond. Exp Hematol Oncol 2013; 2: 17.

16. Liao CL, Herman MM, Bensch KG. Prolongation of G1 and $S$ phase in C-6 glioma cells treated with maple syrup urine disease metabolits. Morphologic and cell cycle studies. Lab Investig J Tech Methods Pathol 1978; 38: 122-133. 
17. Maudsley AA, Gupta RK, Stoyanova R, Parra NA, Roy B, Sheriff S, Hussain N, Behari S. Mapping of glycine distributions in gliomas. AJNR Am J Neuroradiol 2014; 35 (6 Suppl): S31-36.

18. McKnight TR, Smith KJ, Chu PW, Chiu KS, Cloyd CP, Chang SM, Phillips JJ, Berger MS. Choline metabolism, proliferation, and angiogenesis in nonenhancing grades 2 and 3 astrocytoma. J Magn Reson Imaging 2011; 33: 808-816.

19. Metwally LIA, El-din SE, Abdelaziz O, Hamdy IM, Elsamman AK, Abdelalim AM. Predicting grade of cerebral gliomas using Myoinositol/Creatine ratio. Egypt J Radiol Nucl Med 2014; 45: 211-217.

20. Ott D, Hennig J, Ernst T. Human brain tumors: assessment with in vivo proton MR spectroscopy. Radiology 1993; 186: 745-752.

21. Platet N, Liu SY, Atifi ME, Oliver L, Vallette FM, Berger F, Wion D. Influence of oxygen tension on CD133 phenotype in human glioma cell cultures. Cancer Lett 2007; 258: 286-290.

22. Righi V, Andronesi OC, Mintzopoulos D, Black PM, Tzika AA. High-resolution magic angle spinning magnetic resonance spectroscopy detects glycine as a biomarker in brain tumors. Int J Oncol 2010; 36: 301-306.

23. Sabatier J, Gilard V, Malet-Martino M, Ranjeva JP, Terral C, Breil S, Delisle MB, Manelfe C, Tremoulet M, Berry I. Characterization of choline compounds with in vitro $1 \mathrm{H}$ magnetic resonance spectroscopy for the discrimination of primary brain tumors. Invest Radiol 1999; 34: 230-235.

24. Segebarth CM, Balériaux DF, Luyten PR, den Hollander JA. Detection of metabolic heterogeneity of human intracrania tumors in vivo by $1 \mathrm{H}$ NMR spectroscopic imaging. Magn Reson Med 1990; 13: 62-76.

25. Shao W, Gu J, Huang C, Liu D, Huang H, Huang Z, Lin Z, Yang W, Liu K, Lin D, Ji T. Malignancy-associated metabolic profiling of human glioma cell lines using 1 H NMR spectroscopy. Mol Cancer 2014; 13: 197.

26. Stupp R, Mason WP, van den Bent MJ, Weller M, Fisher B, Taphoorn MJ, Belanger K, Brandes AA, Marosi C, Bogdahn U, Curschmann J, Janzer RC, Ludwin SK, Gorlia T, Allgeier A, Lacombe D, Cairncross JG, Eisenhauer E, Mirimanoff RO; European Organisation for Research and Treatment of Cancer Brain Tumor and Radiotherapy Groups; National Cancer Institute of Canada Clinical Trials Group. Radiotherapy plus concomitant and adjuvant temozolomide for glioblastoma. N Engl J Med 2005; 352: 987-996.

27. Tedeschi G, Lundbom N, Raman R, Bonavita S, Duyn JH, Alger JR, Di Chiro G. Increased choline signal coinciding with malignant degeneration of cerebral gliomas: a serial proton magnetic resonance spectroscopy imaging study. J Neurosurg 1997; 87: 516-524.

28. Wang W, Wu Z, Dai Z, Yang Y, Wang J, Wu G. Glycine metabolism in animals and humans: implications for nutrition and health. Amino Acids 2013; 45: 463-477.

29. De Witt Hamer PC, Van Tilborg AA, Eijk PP, Sminia P, Troost D, Van Noorden CJ, Ylstra B, Leenstra S. The genomic profile of human malignant glioma is altered early in primary cell culture and preserved in spheroid spectras s. Oncogene 2008; 27: 2091-2096.

30. Witusik-Perkowska M, Rieske P, Hułas-Bigoszewska K, Zakrzewska M, Stawski R, Kulczycka-Wojdala D, Bieńkowski M, Stoczyńska-Fidelus E, Grešner SM, Piaskowski S, Jaskólski DJ, Papierz W Zakrzewski K, Kolasa M, Ironside JW, Liberski PP. Glioblastoma-derived spheroid cultures as an experimental model for analysis of EGFR anomalies. J Neurooncol 2011; 102: 395-407. 